\title{
Association Analysis of LEP Signaling Pathway with Type 2 Diabetes Mellitus in Chinese Han Population from South China
}

\author{
Haibing Yu (D), Lin Xu, Hao Liu (D), Jialu Huang, Ling Luo, Rong Chen, Jian Xu, \\ Chunwen Lin $\mathbb{D}^{\circ}$, Weiying Chen $\mathbb{D}$, Yuxun Xie, Huihuang Yang, Danli Kong $\mathbb{D}$, \\ and Yuanlin Ding
}

School of Public Health, Guangdong Medical University, Dongguan 523808, China

Correspondence should be addressed to Danli Kong; kdlgdmu@gdmu.edu.cn and Yuanlin Ding; gdmusbd@gdmu.edu.cn

Received 8 January 2021; Revised 3 June 2021; Accepted 20 July 2021; Published 20 September 2021

Academic Editor: Xiang Zhou

Copyright (C) 2021 Haibing Yu et al. This is an open access article distributed under the Creative Commons Attribution License, which permits unrestricted use, distribution, and reproduction in any medium, provided the original work is properly cited.

Objective. This study is aimed at analyzing the relationship between leptin (LEP) signaling pathway and type 2 diabetes mellitus (T2DM) and at providing support for molecular genetic research on the pathogenesis of T2DM in Chinese Han population. Methods. A case-control study was designed, including 1092 cases with T2DM and 1092 healthy controls of Chinese Han origin recruited from ten hospitals in Guangdong Province, Southern China. Twenty-three single nucleotide polymorphisms (SNPs) of 15 genes in LEP signaling pathway were genotyped by SNPscan ${ }^{\mathrm{TM}}$ kit. The Pearson chi-square test, CochranArmitage trend test, MAX3, and logistic regression were applied to analyze the association between single nucleotide polymorphism (SNP) and T2DM; unconditional logistic regression was used to analyze haplotype in LD block; and SNP set analysis based on logistic kernel machine regression was used to analyze pathway. All statistical analysis was performed by SPSS25.0, R2.14, Haploview4.2, SNPStats, and other statistical software packages. Results. In association analysis based on SNP, rs2167270 had statistical significance both in the adjusted and unadjusted covariate dominant model and in the unadjusted covariate overdominant model while it had no significant difference in the adjusted covariate overdominant model. Compared to GG genotype, rs2167270 of AG genotype had statistical significance in both the adjusted and unadjusted covariate codominant models. And rs16147 had statistical significance in robust test, stealth model and overdominant model, and adjusting and unadjusting covariate. This study found linkage disequilibrium existed between rs2167270 and rs4731426 of LEP, rs10889502 and rs17127107 of JAK1, rs2970847 and rs6821591 of PPARGC1A, rs249429 and rs3805486 of PRKAA1, rs1342382 and rs6588640 of PRKAA2, rs3766522 and rs6937 of PRKAB2, rs2970847 and rs6821591 of PRKAG2, and rs6436094 and rs645163 of PRKAG3. There was no positive finding with statistical significance from the unconditional logistic regression of the mentioned genes' haplotype of LD block. Conclusions. LEP signaling pathway association with T2DM remained to be confirmed in Chinese Han population, although rs2167270 and rs16147 were significantly associated with T2DM.

\section{Introduction}

Diabetes has become a major public problem around the world [1], with the numbers increasing from 108 million in 1980 to 422 million in 2014 [2], and the number of people with diabetes is expected to increase to 592 million by 2035 [3]. Type 2 diabetes mellitus (T2DM) is the most prevalent form of diabetes mellitus (DM), accounting for about 90$95 \%$ of the total number of DM cases worldwide [4].
Insulin resistance is the basic pathophysiological change of type 2 diabetes, almost throughout the entire process of the occurrence and development of type 2 diabetes [5]. Studies have shown that free fatty acid (FFA) and adipocytokines released by adipose tissue can cause insulin resistance through a variety of ways [6-9].

For a long time, it has been believed that adipose tissue is only involved in the body's energy storage and nontrembling thermogenesis. With the discovery of leptin (LEP) in 1994 
[10], a series of new functions of adipose tissue were revealed. Adipose tissue is not only an energy storage organ of the body but also an endocrine organ [11]. It is known that human adipocytes secrete dozens of adipocytokines and protein factors, such as leptin, adiponectin, resistin, and visfatin. Some of these adipocytokines enter the human blood circulation to regulate the functions of distant target organs, and the other part plays a role of adjacent secretion or autocrine regulation in adjacent tissues or cells or within cells.

Leptin is a secreted protein synthesized by fat cells. It is the coded product of obese gene (ob gene). It exists freely in the blood or binds to leptin-binding protein. It has a wide range of centers such as reducing food intake and increasing energy consumption. And peripheral tissue effects are mediated through leptin receptors [12]. Leptin receptors belong to the class I cytokine receptor family and are widely distributed in central and peripheral tissues. Recent research results have shown that the occurrence and development of obesity, insulin resistance, and type 2 diabetes are closely related to the abnormal function of adipocytokines or their receptors [13-15]. Leptin can cause insulin resistance and hyperinsulinemia through a variety of ways, and hyperinsulinemia and insulin resistance can cause excessive secretion of leptin, thus forming a vicious circle, leading to abnormal glucose and lipid metabolism $[12,16]$.

Single nucleotide polymorphism mainly refers to the polymorphism of DNA sequence caused by the variation of single nucleotide at the genome level. It is the most common type of human heritable variation. Recent studies have shown that there is an association between SNPs in multiple genes and T2DM and its complications. Letonja et al.'s [17] group finds a significant association between the SIRT1 rs7069102 polymorphism and diabetic nephropathy caused by T2DM. Zhang et al.'s [18] group discovers that rs62099905 in the CNDP1 gene was related to the serum glucose level of healthy Chinese Han population. Cui et al.'s [19] study shows that ADIPOQ gene polymorphisms rs10937273, rs1501299, rs182052, rs2241767, and rs266729 are associated with type 2 diabetes. Zhu et al.'s [20] study suggests that the interaction effects of SNPSNP and SNP-environmental factors were related to T2DM susceptibility, and Khan et al.'s [21] research shows that the genetic variation of the TCF7L2 gene is related to the increased susceptibility to T2DM. Juttada et al.'s [22] group discovers that TCF7L2 gene polymorphism is closely related to a positive family history of diabetes. Bains et al.'s [23] study suggests that LEPrs7799039 and LEPRrs1137101 polymorphism conferred 3.4- and 2.1-fold risk towards the development of T2DM after adjustment of various covariates under a recessive genetic model.

This study intends to analyze the association between a SNP and type 2 diabetes based on the LEP signaling pathway, hoping to discover new mutation sites that may cause insulin resistance, conduct linkage disequilibrium analysis and haplotype-based association analysis, and analyze multiple loci of a single gene. Evaluate the distribution difference of haplotypes formed by points between the case group and the control group; conduct SNP-SNP interaction analysis to find meaningful interaction pairs; use pathway analysis to explore the relationship between LEP signaling pathway and T2DM.

\section{Materials and Methods}

2.1. Study Population. During November 2011 to October 2013, case-control groups of 1092 patients diagnosed as having T2DM were recruited from 10 hospitals in Zhanjiang, Maoming, Shaoguan, Dongguan, and Shenzhen of Guangdong Province. T2DM was diagnosed according to the criteria of the World Health Organization (WHO) in 1999. The inclusive criteria of the T2DM patient group were as follows: (1) range of ages 20-70; (2) random blood glucose levels $\geq 11.1 \mathrm{mmol} / \mathrm{l}$ with diabetes symptoms including polydipsia, polyphagia, polyuria, weight loss, itchiness, blurred vision, and other acute metabolic disorders caused by hyperglycemia and fasting plasma glucose levels $\geq 7.0 \mathrm{mmol} / \mathrm{l}$ without diabetes symptoms or blood glucose levels $\geq 11.1$ mmol/l with a glucose tolerance test after two hours oral dose; and (3) patients without other serious diseases, such as cardiovascular and cerebrovascular diseases, malignant tumors, and other diseases. The control group comprised 1092 normal persons who were examined in the same hospital at the same time as the cases. The inclusive criteria of the control group were as follows: (1) range of ages 20-70; (2) without a family history of diabetes; and (3) healthy after physical examination including medical history, blood glucose, and other biochemical test results. This study was approved by the Ethics Committee. All the surveys and samples obtained the consents of participants in advance, and the informed consent forms had been legally consented. All the participants were the permanent residents of Chinese Han nationality in Guangdong Province, and there is no blood relationship among them.

\subsection{Information Collection and Blood Sample Collection. A} standardized survey is aimed at respondents who met the inclusion criteria, including age, gender, place of origin, occupation, disease history, duration of illness, smoking history, family history, complications, and diet, and exercise was conducted by a uniformly trained investigator. At the end of the investigation, the height and weight of the subjects were measured by a height and weight scale, and the blood pressure of the right arm in a sitting position was measured with a benchtop mercury sphygmomanometer, twice, and averaged.

$5 \mathrm{ml}$ of peripheral blood was collected by the endocrine nurses in the morning, and the relative clinical biochemical indicators, including fasting plasma glucose (FPG), total cholesterol (TC), triglyceride (TG), high-density lipoprotein cholesterol (HDL-C), low-density lipoprotein cholesterol (LDL-C), and glycosylated hemoglobin A1c (HbA1c), were detected. The FPG level was measured by the glycosylation method, and the plasma levels of TC, TG, HDL-C, and LDL-C were determined in an enzymatic method. HBA1C was determined by a high-performance liquid chromatography (BIO-RADD-10TM glycated hemoglobin detection system), and other biochemical indicators were detected by an automatic biochemical analyzer. Besides, $4 \mathrm{ml}$ of peripheral 
TABLE 1: Basic information of 23 tagSNPs selected from 15 genes in the LEP signaling pathway.

\begin{tabular}{|c|c|c|c|c|c|c|c|}
\hline \multirow{2}{*}{ Gene } & \multirow{2}{*}{ Chr } & \multirow{2}{*}{ Position_37 } & \multirow{2}{*}{ SNP } & \multirow{2}{*}{ Region } & \multicolumn{2}{|c|}{ Allele } & \multirow{2}{*}{ MAF } \\
\hline & & & & & Minor & Major & \\
\hline \multirow[t]{2}{*}{ LEP } & 7 & 127882070 & rs4731426 & Intron 1 & G & $\mathrm{C}$ & 0.202 \\
\hline & 7 & 127881349 & $\mathrm{rs} 2167270$ & $5^{\prime} \mathrm{UTR}$ & G & A & 0.175 \\
\hline LEPR & 1 & 66063117 & rs 12405556 & Intron 7 & G & $\mathrm{T}$ & 0.153 \\
\hline \multirow{2}{*}{ JAK1 } & 1 & 65326908 & rs 17127107 & Intron 8 & $\mathrm{C}$ & G & 0.321 \\
\hline & 1 & 65379982 & rs 10889502 & Intron 1 & G & $\mathrm{C}$ & 0.375 \\
\hline JAK2 & 9 & 4988761 & rs7849191 & Intron 2 & $\mathrm{C}$ & $\mathrm{T}$ & 0.420 \\
\hline STAT3 & 17 & 40507980 & rs9891119 & Intron 1 & A & $\mathrm{C}$ & 0.357 \\
\hline SHP-2 & 12 & 112942353 & rs4767860 & Intron 14 & G & A & 0.409 \\
\hline \multirow[t]{2}{*}{ PPARGC1A } & 4 & 23815924 & rs2970847 & Exon 8 & $\mathrm{~T}$ & $\mathrm{C}$ & 0.204 \\
\hline & 4 & 23797000 & rs6821591 & $3^{\prime} \mathrm{UTR}$ & $\mathrm{C}$ & $\mathrm{T}$ & 0.292 \\
\hline \multirow[t]{2}{*}{ PRKAA1 } & 5 & 40782239 & rs249429 & Intron 1 & $\mathrm{C}$ & $\mathrm{T}$ & 0.255 \\
\hline & 5 & 40796045 & rs3805486 & Intron 1 & A & G & 0.212 \\
\hline \multirow[t]{2}{*}{ PRKAA2 } & 1 & 57177388 & rs1342382 & $3^{\prime} \mathrm{UTR}$ & $\mathrm{T}$ & A & 0.252 \\
\hline & 1 & 57108423 & rs6588640 & $5^{\prime}$ upstream & A & G & 0.168 \\
\hline \multirow[t]{2}{*}{ PRKAB2 } & 1 & 146626922 & rs6937 & $3^{\prime}$ UTR & $\mathrm{T}$ & $\mathrm{C}$ & 0.409 \\
\hline & 1 & 146641686 & rs3766522 & Intron 2 & A & $\mathrm{T}$ & 0.128 \\
\hline PRKAG1 & 12 & 49390677 & rs10783299 & Exon 2 & $\mathrm{~T}$ & $\mathrm{C}$ & 0.346 \\
\hline \multirow[t]{2}{*}{ PRKAG2 } & 7 & 151255203 & rs5017427 & Intron 15 & A & G & 0.261 \\
\hline & 7 & 151502302 & rs9648724 & Intron 1 & G & A & 0.245 \\
\hline \multirow[t]{2}{*}{ PRKAG3 } & 2 & 219682257 & rs645163 & $3^{\prime}$ downstream & $\mathrm{C}$ & $\mathrm{T}$ & 0.416 \\
\hline & 2 & 219687597 & rs6436094 & $3^{\prime} \mathrm{UTR}$ & A & G & 0.444 \\
\hline Alpha-MSH & 2 & 25384833 & rs6713532 & Intron 2 & $\mathrm{~T}$ & $\mathrm{C}$ & 0.374 \\
\hline NPY & 7 & 24323410 & rs16147 & $5^{\prime}$ upstream & $\mathrm{T}$ & $\mathrm{C}$ & 0.332 \\
\hline
\end{tabular}

blood of the subjects ( $2 \mathrm{ml}$ per tube) was collected, anticoagulated with EDTA.k2, and stored at $-80^{\circ} \mathrm{C}$.

This study was approved by the ethics committee, and all the investigations and sampling were subject to the consent of the subjects with signed informed consent.

2.3. Data Collation and Database Establishment. All completed questionnaires were uniformly coded, and all participants' questionnaire information, physical examination, and clinical biochemical examination results were compiled. We used EpiData3.1 software to build a database with data by double input. The entered data was checked by both manual and computer methods to ensure that the data has no logic errors and no entry errors.

\subsection{DNA Extraction}

(1) Transfer the blood sample from the anticoagulation tube to a $10 \mathrm{ml}$ centrifuge tube, and mark the centrifuge tube

(2) Add $2 \mathrm{ml}$ of cell lysate CL to blood containing anticoagulant, mix upside down, centrifuge at $5000 \mathrm{rpm}$ for $10 \mathrm{~min}$, and discard the supernatant

(3) Add $3 \mathrm{ml}$ of cell lysate CL to it, mix upside down, centrifuge at $5000 \mathrm{rpm}$ for $10 \mathrm{~min}$, discard the
TABLE 2: Comparison of baseline data between the case group and control group.

\begin{tabular}{lccc}
\hline Parameters & T2DM & Control & $P$ \\
$n$ & 1067 & 1054 & - \\
\hline Male (\%) & $532(49.86)$ & $532(50.47)$ & 0.080 \\
Age $($ years $)$ & $59.71 \pm 11.87$ & $57.23 \pm 10.41$ & $<0.001$ \\
BMI $\left(\mathrm{kg} / \mathrm{m}^{2}\right)$ & $24.60 \pm 3.24$ & $23.58 \pm 3.33$ & $<0.001$ \\
FPG $(\mathrm{mmol} / \mathrm{l})$ & $10.46 \pm 4.50$ & $5.60 \pm 1.60$ & $<0.001$ \\
TC $(\mathrm{mmol} / \mathrm{l})$ & $5.31 \pm 1.59$ & $5.43 \pm 1.27$ & 0.056 \\
Triglyceride $(\mathrm{mmol} / \mathrm{l})$ & $2.24 \pm 1.03$ & $1.31 \pm 0.96$ & $<0.001$ \\
HDL-C (mmol/l) & $1.35 \pm 0.54$ & $1.37 \pm 0.42$ & 0.398 \\
LDL-C (mmol/l) & $2.73 \pm 1.04$ & $3.03 \pm 0.65$ & $<0.001$ \\
Hypertension $(\%)$ & $396(37.11)$ & $380(36.05)$ & 0.257 \\
Heart rate & $76.40 \pm 15.26$ & $76.20 \pm 10.92$ & 0.682 \\
\hline
\end{tabular}

BMI (body mass index $)=$ body weight $/$ (height $*$ height $)$.

supernatant, and put the centrifuge tube upside down on clean absorbent paper for 1 min and dry by airing

(4) Configure a mixture of buffer FG and proteinase $\mathrm{K}$ ( $1 \mathrm{ml}$ buffer $+10 \mu \mathrm{l}$ proteinase $\mathrm{K}$, ready for use) 
TABLE 3: 23SNP genotyping results for 15 genes in the LEP signaling pathway.

\begin{tabular}{|c|c|c|c|c|c|c|}
\hline \multirow{2}{*}{ Gene } & \multirow{2}{*}{ SNP } & \multicolumn{2}{|c|}{ Allele } & \multirow{2}{*}{ Call rate $(\%)$} & \multirow{2}{*}{ MAF } & \multirow{2}{*}{$P_{\text {HWE }}$} \\
\hline & & Minor & Major & & & \\
\hline \multirow[t]{2}{*}{ LEP } & rs4731426 & $\mathrm{G}$ & $\mathrm{C}$ & 98.37 & 0.303 & 0.161 \\
\hline & rs2167270 & A & G & 98.34 & 0.238 & 0.095 \\
\hline LEPR & rs12405556 & G & $\mathrm{T}$ & 98.34 & 0.158 & 1.002 \\
\hline \multirow[t]{2}{*}{ JAK1 } & rs17127107 & $\mathrm{C}$ & G & 98.32 & 0.314 & 0.152 \\
\hline & rs10889502 & G & $\mathrm{C}$ & 98.37 & 0.340 & 0.943 \\
\hline JAK2 & rs7849191 & $\mathrm{C}$ & $\mathrm{T}$ & 98.37 & 0.369 & 0.351 \\
\hline STAT3 & rs9891119 & A & $\mathrm{C}$ & 98.37 & 0.445 & 0.851 \\
\hline SHP-2 & rs4767860 & G & A & 98.24 & 0.347 & 0.842 \\
\hline \multirow[t]{2}{*}{ PPARGC1A } & rs2970847 & $\mathrm{T}$ & C & 98.37 & 0.241 & 0.153 \\
\hline & rs6821591 & $\mathrm{C}$ & $\mathrm{T}$ & 98.37 & 0.328 & 0.672 \\
\hline \multirow[t]{2}{*}{ PRKAA1 } & rs249429 & $\mathrm{C}$ & $\mathrm{T}$ & 98.37 & 0.220 & 0.473 \\
\hline & rs3805486 & $\mathrm{A}$ & G & 98.34 & 0.234 & 0.111 \\
\hline \multirow[t]{2}{*}{ PRKAA2 } & rs1342382 & $\mathrm{T}$ & A & 98.34 & 0.253 & 0.332 \\
\hline & rs6588640 & $\mathrm{A}$ & G & 98.37 & 0.251 & 0.574 \\
\hline \multirow[t]{2}{*}{ PRKAB2 } & rs6937 & $\mathrm{T}$ & $\mathrm{C}$ & 98.34 & 0.451 & 0.421 \\
\hline & rs3766522 & $\mathrm{A}$ & $\mathrm{T}$ & 98.37 & 0.119 & 0.642 \\
\hline PRKAG1 & rs10783299 & $\mathrm{T}$ & $\mathrm{C}$ & 98.34 & 0.474 & 0.622 \\
\hline \multirow[t]{2}{*}{ PRKAG2 } & rs5017427 & A & G & 98.37 & 0.290 & 0.563 \\
\hline & rs9648724 & G & $\mathrm{A}$ & 98.29 & 0.235 & 0.353 \\
\hline \multirow[t]{2}{*}{ PRKAG3 } & rs645163 & $\mathrm{C}$ & $\mathrm{T}$ & 98.37 & 0.422 & 0.902 \\
\hline & rs6436094 & $\mathrm{A}$ & G & 98.37 & 0.450 & 0.803 \\
\hline Alpha-MSH & rs6713532 & $\mathrm{T}$ & $\mathrm{C}$ & 98.37 & 0.411 & 0.056 \\
\hline NPY & rs16147 & $\mathrm{T}$ & $\mathrm{C}$ & 98.24 & 0.362 & 0.0051 \\
\hline
\end{tabular}

(5) Add $1 \mathrm{ml}$ of mixed solution to each sample and vortex immediately until the solution is free of clumps

(6) Perform water bath at $65^{\circ} \mathrm{C}$ for 20 minutes, slowly tilting and inverting and mixing several times during this period

(7) Add $1 \mathrm{ml}$ of isopropanol, and mix thoroughly by inversion until filaments or clusters (DNA) appear

(8) Centrifuge at $5000 \mathrm{rpm}$ for 15 minutes, discard the supernatant, and place the centrifuge tube upside down on a clean absorbent paper to ensure that the precipitate is in the tube

(9) Add $1 \mathrm{ml}$ of $70 \%$ ethanol, transfer to a $1.5 \mathrm{ml}$ centrifuge tube, centrifuge at $8000 \mathrm{rpm}$ for $10 \mathrm{~min}$ in a small centrifuge, and discard the supernatant

(10) Add $1 \mathrm{ml}$ of $70 \%$ ethanol, centrifuge at $8000 \mathrm{rpm}$ for $10 \mathrm{~min}$ in a small centrifuge, and discard the supernatant

(11) Dry the DNA pellet in the centrifuge tube at room temperature until the liquid evaporates

(12) Add the corresponding amount of buffer TB according to the amount of DNA; heat at $65^{\circ} \mathrm{C}$ for 1 hour to dissolve the DNA, during which time the finger flicks several times to aid the solution; and finally centrifuge for $30 \mathrm{~s}$ to shake the undissolved precipitate to the bottom of the tube

(13) Perform DNA quality detection with a nucleic acid protein content detector, record DNA concentration $(\mathrm{ng} / \mu \mathrm{l})$ and purity (OD260/280 value, OD260/230 value), and finally store the DNA in a refrigerator at $-80^{\circ} \mathrm{C}$

2.5. Screening and Typing of SNP. The pathway diagram of LEP signaling pathway was obtained from the KEGG database (Kyoto Encyclopedia of Genes and Genomes), and 15 main genes were selected, including LEP, LEPR, JAK (JAK1, JAK2), STAT3, SHP-2 (PTPN11), PPARGC1Alpha (PPARGC1A), AMPK (PRKAA1, PRKAA2, PRKAB2, PRKAG1, PRKAG2, and PRKAG3), alpha-MSH (POMC), and NPY. Haploview (ver.4.2) was used to select tagSNPs from selected genes and their upstream and downstream $5 \mathrm{~kb}$ regions, requiring $\mathrm{MAF}$ (minor allele frequency) $>0.05$ and $r^{2} \geq 0.8$ (SNP MAF and LD data come from the HapMap database, release 27, http://snp.cshl.org/). Then, use FastSNP (http://fastsnp.ibms.sinica.edu.tw/pages/input

CandidateGeneSearch.jsp) to predict the potential function of tagSNP, select tagSNP with higher prediction score, and select 1-2 tagSNP for each gene. Finally, 23 SNPs were selected from 15 genes. These SNPs were distributed on 8 chromosomes, one of which was located downstream of the 
TABLE 4: Results of association analysis between LEP signaling pathway allele and type2 diabetes.

\begin{tabular}{|c|c|c|c|c|c|c|c|c|c|c|c|c|}
\hline \multirow[t]{2}{*}{ Gene } & \multirow[t]{2}{*}{ SNP } & \multirow[t]{2}{*}{ Allele } & \multicolumn{3}{|c|}{$\begin{array}{l}\text { Nondiabetic } \\
\text { controls }\end{array}$} & \multicolumn{3}{|c|}{ T2DM patients } & \multicolumn{2}{|c|}{$P$ value } & \multicolumn{2}{|c|}{ OR $(95 \% \mathrm{CI})$} \\
\hline & & & A & $\mathrm{a}$ & MAF & A & $\mathrm{a}$ & MAF & Observed & Adjusted & Observed & Adjusted \\
\hline \multirow[t]{2}{*}{ LEP } & rs4731426 & $\mathrm{C} / \mathrm{G}$ & 1488 & 620 & 0.294 & 1460 & 674 & 0.316 & 0.132 & 0.141 & $1.11(0.97-1.26)$ & $1.11(0.97-1.26)$ \\
\hline & rs 2167270 & G/A & 1630 & 478 & 0.227 & 1599 & 535 & 0.251 & 0.071 & 0.079 & $1.14(0.99-1.31)$ & $1.14(0.99-1.31)$ \\
\hline LEPR & rs12405556 & $\mathrm{T} / \mathrm{G}$ & 1775 & 333 & 0.158 & 1802 & 332 & 0.156 & 0.833 & 0.850 & $0.98(0.83-1.16)$ & $0.98(0.83-1.16)$ \\
\hline \multirow[t]{2}{*}{ JAK1 } & rs17127107 & $\mathrm{C} / \mathrm{G}$ & 1454 & 654 & 0.310 & 1451 & 683 & 0.320 & 0.491 & 0.610 & $1.05(0.92-1.20)$ & $1.04(0.91-1.18)$ \\
\hline & rs10889502 & $\mathrm{C} / \mathrm{G}$ & 1400 & 708 & 0.336 & 1385 & 749 & 0.351 & 0.310 & 0.292 & $1.07(0.94-1.22)$ & $1.07(0.94-1.22)$ \\
\hline JAK2 & rs7849191 & $\mathrm{C} / \mathrm{T}$ & 1330 & 778 & 0.369 & 1349 & 785 & 0.368 & 0.932 & 0.963 & $0.99(0.88-1.13)$ & $1.00(0.88-1.13)$ \\
\hline STAT3 & rs9891119 & $\mathrm{A} / \mathrm{C}$ & 1170 & 938 & 0.445 & 1159 & 975 & 0.457 & 0.431 & 0.451 & $1.05(0.93-1.18)$ & $1.05(0.93-1.19)$ \\
\hline SHP-2 & rs4767860 & $\mathrm{A} / \mathrm{G}$ & 1401 & 705 & 0.335 & 1390 & 742 & 0.348 & 0.360 & 0.334 & $1.06(0.93-1.21)$ & $1.07(0.94-1.22)$ \\
\hline \multirow[t]{2}{*}{ PPARGC1A } & rs2970847 & $\mathrm{C} / \mathrm{T}$ & 1604 & 504 & 0.239 & 1623 & 511 & 0.239 & 0.982 & 0.872 & $1.00(0.87-1.15)$ & $1.01(0.88-1.16)$ \\
\hline & rs6821591 & $\mathrm{T} / \mathrm{C}$ & 1419 & 689 & 0.327 & 1442 & 692 & 0.324 & 0.863 & 0.782 & $0.99(0.87-1.12)$ & $0.98(0.86-1.12)$ \\
\hline \multirow[t]{2}{*}{ PRKAA1 } & rs249429 & $\mathrm{T} / \mathrm{C}$ & 1654 & 454 & 0.215 & 1652 & 482 & 0.226 & 0.411 & 0.543 & $1.06(0.92-1.23)$ & $1.05(0.90-1.21)$ \\
\hline & rs3805486 & $\mathrm{A} / \mathrm{G}$ & 1608 & 500 & 0.237 & 1642 & 492 & 0.231 & 0.614 & 0.961 & $0.96(0.84-1.11)$ & $1.00(0.86-1.15)$ \\
\hline \multirow[t]{2}{*}{ PRKAA2 } & rs 1342382 & $\mathrm{~T} / \mathrm{A}$ & 1567 & 541 & 0.257 & 1598 & 536 & 0.251 & 0.680 & 0.721 & $0.97(0.84-1.12)$ & $0.97(0.85-1.12)$ \\
\hline & rs6588640 & G/A & 1567 & 541 & 0.257 & 1611 & 523 & 0.245 & 0.392 & 0.373 & $0.94(0.82-1.08)$ & $0.94(0.81-1.08)$ \\
\hline \multirow[t]{3}{*}{ PRKAB2 } & rs6937 & $\mathrm{C} / \mathrm{T}$ & 1167 & 941 & 0.446 & 1131 & 1002 & 0.470 & 0.123 & 0.272 & $1.10(0.97-1.25)$ & $1.07(0.95-1.22)$ \\
\hline & rs3766522 & $\mathrm{A} / \mathrm{T}$ & 1875 & 233 & 0.111 & 1864 & 270 & 0.127 & 0.112 & 0.241 & $1.17(0.97-1.41)$ & $1.12(0.93-1.36)$ \\
\hline & rs10783299 & $\mathrm{T} / \mathrm{C}$ & 1114 & 994 & 0.472 & 1152 & 980 & 0.460 & 0.451 & 0.531 & $0.95(0.85-1.08)$ & $0.96(0.85-1.09)$ \\
\hline \multirow[t]{2}{*}{ PRKAG2 } & rs5017427 & G/A & 1475 & 633 & 0.300 & 1541 & 593 & 0.278 & 0.112 & 0.130 & $0.90(0.79-1.02)$ & $0.90(0.79-1.03)$ \\
\hline & rs9648724 & G/A & 1605 & 503 & 0.239 & 1621 & 511 & 0.240 & 0.933 & 0.990 & $1.01(0.87-1.16)$ & $1.00(0.86-1.16)$ \\
\hline \multirow[t]{2}{*}{ PRKAG3 } & rs645163 & $\mathrm{C} / \mathrm{T}$ & 1211 & 897 & 0.426 & 1205 & 929 & 0.435 & 0.521 & 0.654 & $1.04(0.92-1.17)$ & $1.03(0.91-1.16)$ \\
\hline & rs6436094 & G/A & 1175 & 933 & 0.443 & 1196 & 938 & 0.440 & 0.840 & 0.992 & $0.99(0.88-1.11)$ & $1.00(0.88-1.13)$ \\
\hline Alpha-MSH & rs6713532 & $\mathrm{C} / \mathrm{T}$ & 1241 & 867 & 0.411 & 1257 & 877 & 0.411 & 0.982 & 0.922 & $1.00(0.88-1.13)$ & $1.01(0.89-1.14)$ \\
\hline NPY & rs16147 & $\mathrm{C} / \mathrm{T}$ & 1343 & 765 & 0.363 & 1382 & 748 & 0.351 & 0.432 & 0.331 & $0.95(0.84-1.08)$ & $0.94(0.83-1.07)$ \\
\hline
\end{tabular}

$3^{\prime}$ end, 4 of which were located in the untranslated region of the $3^{\prime}$ end, 2 of which were located upstream of the $5^{\prime}$ end, one of which was located in the $5^{\prime}$ untranslated region, 13 of which were located in the intron region, and 2 of which were located in the exon region. In the Chinese population, the minimum MAF was 0.128 (rs3766522) and the maximum MAF was 0.444 (rs6436094). Related information is listed in Table 1.

2.6. Statistical Analysis. Comparisons of all variables between T2DM cases and control subjects were carried out by the $\chi^{2}$ test for categorical variables or $t$-test for continuous variables. The Pearson chi-square test, Cochran-Armitage trend test, MAX3, and logistic regression were used to analyze the association between SNP and T2DM; unconditional logistic regression was used to analyze haplotype in LD block; and SNP set analysis based on logistic kernel machine regression was used to analyze pathway. All statistical analysis was performed by SPSS25.0, PLINK 1.07, R 2.14.2, Haploview 4.2, SNPStats, and other statistical software packages.

\section{Results}

3.1. Equilibrium Test Results of Baseline Data in the Case Group and Control Group. In this study, 1092 cases and 1092 controls were included, and the individuals with the loss rate of SNP type $>20 \%$ were excluded. Eventually, 1067 cases and 1054 controls were included in the following analysis, including 532 males and 535 females in the case group with an average age of 49.86 years and 532 males and 522 females in the control group with an average age of 50.47 years. Differences of the age composition, body mass index (BMI), fasting plasma glucose (FPG), triglyceride level, and lowdensity cholesterol level between the two groups were statistically significant. The average age, BMI, FPG, and triglyceride level of the case group are higher than those of the control group, as shown in Table 2.

3.2. SNP Typing Results. The typing success rate of 23 SNPs was all above $98 \%$, and the minimum allele frequency was 0.119 , and the maximum was 0.474 . The Hardy-Weinberg equilibrium test shows that each point satisfies the HardyWeinberg equilibrium, as shown in Table 3.

3.3. Allele Association Analysis Results. The results of genotype association analysis are shown in Table 4. There was no statistical difference in the suballelic frequency of each SNP between the case group and the control group $(P>0.05)$. After adjusting for covariates such as age and BMI, there was no statistical difference in the suballelic frequency between the case group and the control group. 
TABLE 5: Comparison of genotype frequencies between the case group and control group in the LEP signaling pathway.

\begin{tabular}{|c|c|c|c|c|c|c|}
\hline \multirow{2}{*}{ Gene } & \multirow{2}{*}{ SNP } & \multirow{2}{*}{ Genotype } & \multirow{2}{*}{ Nondiabetic controls } & \multirow{2}{*}{ T2DM patients } & \multicolumn{2}{|c|}{$P$ value } \\
\hline & & & & & $\chi^{2}$ & $P$ value \\
\hline \multirow[t]{2}{*}{ LEP } & rs4731426 & $\mathrm{CC} / \mathrm{CG} / \mathrm{GG}$ & $535 / 418 / 101$ & $499 / 462 / 106$ & 3.495 & 0.174 \\
\hline & rs 2167270 & GG/GA/AA & $640 / 350 / 64$ & $598 / 403 / 66$ & 5.107 & 0.078 \\
\hline LEPR & rs 12405556 & TT/TG/GG & $747 / 281 / 26$ & $763 / 276 / 28$ & 0.209 & 0.901 \\
\hline \multirow[t]{2}{*}{ JAK1 } & rs 17127107 & $\mathrm{CC} / \mathrm{CG} / \mathrm{GG}$ & $491 / 472 / 91$ & $490 / 471 / 106$ & 1.065 & 0.587 \\
\hline & rs 10889502 & $\mathrm{CC} / \mathrm{CG} / \mathrm{GG}$ & $464 / 472 / 118$ & $441 / 503 / 123$ & 1.594 & 0.451 \\
\hline JAK2 & rs7849191 & $\mathrm{CC} / \mathrm{CT} / \mathrm{TT}$ & $412 / 506 / 136$ & $420509 / 138$ & 0.021 & 0.990 \\
\hline STAT3 & rs9891119 & $\mathrm{AA} / \mathrm{AC} / \mathrm{CC}$ & $323 / 524 / 207$ & $315 / 529 / 223$ & 0.640 & 0.726 \\
\hline SHP-2 & rs4767860 & $\mathrm{AA} / \mathrm{AG} / \mathrm{GG}$ & $464 / 473 / 116$ & $452 / 486 / 128$ & 0.844 & 0.656 \\
\hline \multirow[t]{2}{*}{ PPARGC1A } & rs2970847 & $\mathrm{CC} / \mathrm{CT} / \mathrm{TT}$ & $619 / 366 / 69$ & $624 / 375 / 68$ & 0.057 & 0.972 \\
\hline & rs6821591 & TT/TC/CC & $474 / 471 / 109$ & $492 / 458 / 117$ & 0.721 & 0.697 \\
\hline \multirow[t]{2}{*}{ PRKAA1 } & rs249429 & TT/TC/CC & $653 / 348 / 53$ & $639 / 374 / 54$ & 1.018 & 0.601 \\
\hline & rs3805486 & AA/AG/GG & $623 / 362 / 69$ & $633 / 376 / 58$ & 1.218 & 0.544 \\
\hline \multirow[t]{2}{*}{ PRKAA2 } & rs1342382 & TT/TA/AA & $576 / 415 / 63$ & $598 / 402 / 67$ & 0.663 & 0.718 \\
\hline & rs6588640 & GG/GA/AA & $586 / 395 / 73$ & 609/393/65 & 0.832 & 0.660 \\
\hline \multirow[t]{2}{*}{ PRKAB2 } & rs6937 & CC/CT/TT & $316 / 535 / 203$ & $285 / 562 / 220$ & 2.867 & 0.238 \\
\hline & rs3766522 & AA/AT/TT & $832 / 211 / 11$ & $812 / 240 / 15$ & 2.644 & 0.267 \\
\hline PRKAG1 & rs10783299 & TT/TC/CC & $290 / 534 / 230$ & $308 / 536 / 222$ & 0.619 & 0.734 \\
\hline \multirow[t]{2}{*}{ PRKAG2 } & rs5017427 & GG/GA/AA & $520 / 435 / 99$ & $561 / 419 / 87$ & 2.549 & 0.280 \\
\hline & rs9648724 & GG/GA/AA & $605 / 395 / 54$ & $610 / 401 / 55$ & 0.007 & 0.996 \\
\hline \multirow[t]{2}{*}{ PRKAG3 } & rs645163 & $\mathrm{CC} / \mathrm{CT} / \mathrm{TT}$ & $349 / 513 / 192$ & $344 / 517 / 206$ & 0.464 & 0.793 \\
\hline & rs6436094 & GG/GA/AA & $325 / 525 / 204$ & $341 / 514 / 212$ & 0.575 & 0.750 \\
\hline Alpha-MSH & rs6713532 & CC/CT/TT & $350 / 541 / 163$ & $365 / 527 / 175$ & 0.845 & 0.656 \\
\hline NPY & rs16147 & CC/CT/TT & $449 / 445 / 160$ & $442 / 498 / 125$ & 7.275 & 0.026 \\
\hline
\end{tabular}

3.4. Genotype Association Analysis Results. The genotype (CC/CT/TT) distribution of rs16147 was statistically significant between the two groups $\left(\chi^{2}=7.275, P=0.026\right)$. There was no statistical difference in genotype distribution of other SNPs, as shown in Table 5.

In order to further confirm whether each SNP was associated with T2DM and whether the incidence of disease increases with the increase of risk alleles in the genotype, we conducted the Cochran-Armitage trend test under different genetic models (additive model, codominant model, dominant model, invisible model, and superdominant model), and the results are shown in Table 6.

Since the trend test relies on prespecified scores, different scores correspond to different genetic models. Only when the designated gene model is a true gene model the corresponding trend test is the test with the best power. We further applied the robust test method and compared the results obtained with the methods based on various genetic models (see Table 7 for details). The result of rs16147 was statistically significant $(P=0.045)$. The other SNPs were not statistically significant in the robust test results.

After adjusting the covariate, the results under the five genetic models are shown in Table 8. rs2167270 still has statistical significance in the dominant model $\left(P_{\text {adj }}=0.039\right.$, OR $=1.20,95 \%$ CI: 1.011 .44$)$, while there is no statistical significance in the superdominant model; in the codominant model, compared to GG genotype, AG genotype $\left(\mathrm{OR}=1.22,95 \% \mathrm{CI}: 1.011 .47, P_{\text {adj }}=0.024\right.$ ) is statistically significant, and compared to GG genotype, AA genotype $\left(\mathrm{OR}=1.12,95 \% \mathrm{CI}: 0.781 .63, P_{\text {adj }}\right.$ $=0.028)$ is still not statistically significant. rs16147 was still statistically significant in both the stealth model and the superdominant model, in the recessive model $\left(P_{\text {adj }}=0.012\right.$, OR $\left.=0.72,95 \% \mathrm{CI}: 0.56-0.93\right)$, and in the superdominant model $\left(P_{\mathrm{adj}}=0.044, \mathrm{OR}=1.21,95 \% \mathrm{CI}\right.$ : 1.01-1.44).

3.5. Linkage Disequilibrium Analysis and Association Analysis Based on Haplotype. Linkage is a genetic tendency where genetic markers are inherited together as a result of being near to one another on the same chromosome. Genetic linkage analysis, one of the old study approaches, focuses on genomic regions with large genetic effect that can influence the development of a disease [24, 25].

Haploview 4.2 software was used to analyze the linkage imbalance (LD) between different loci in the same gene, and the results showed that there was linkage imbalance between loci in 8 genes including LEP. Figure 1 shows the composition of LD block of 8 genes including LEP. 


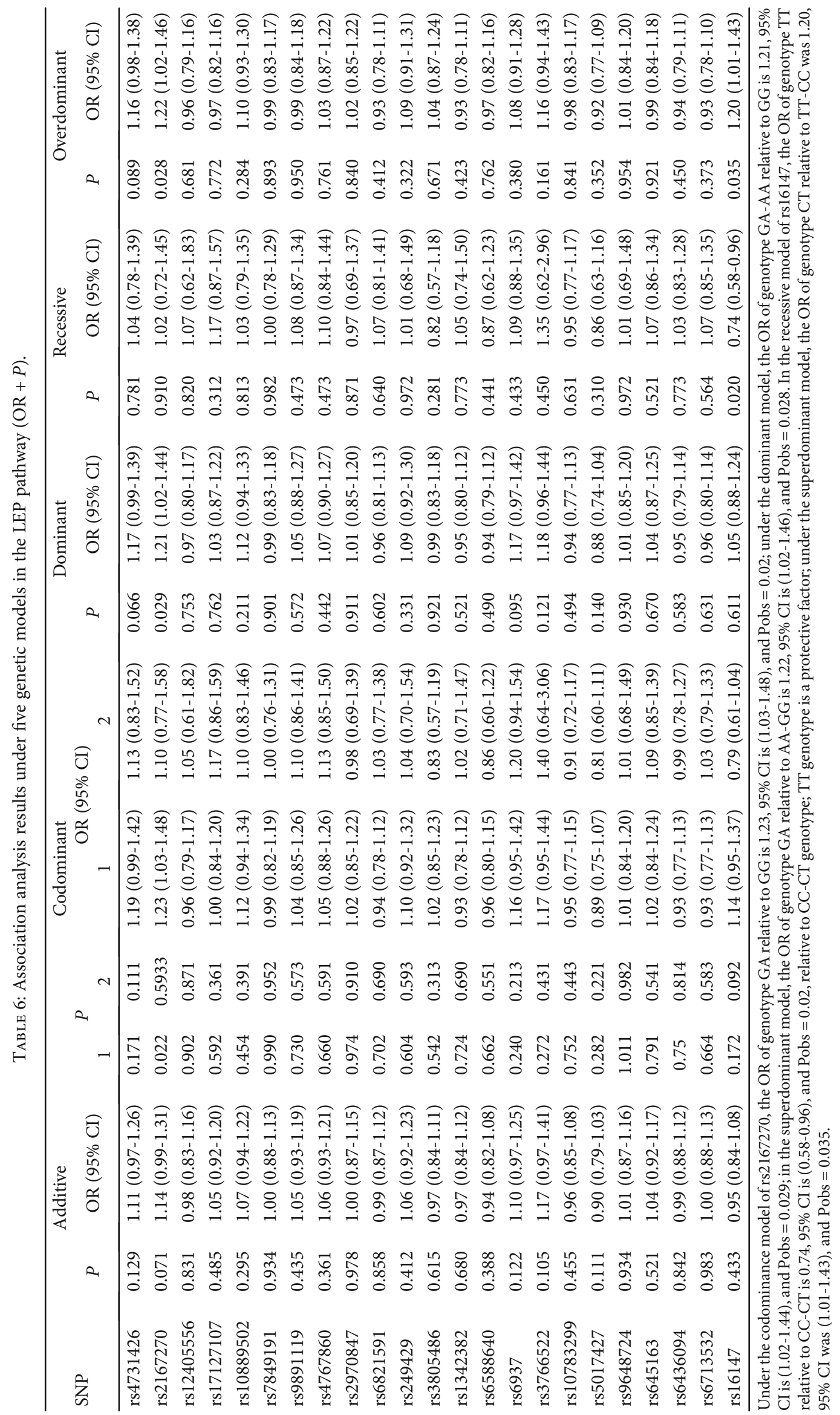


TABLE 7: Robustness test results of the MAX3 method.

\begin{tabular}{lccc}
\hline Gene & SNP & $\chi^{2}$ & $P$ \\
\hline LEP & rs4731426 & 1.839 & 0.138 \\
LEPR & rs2167270 & 2.184 & 0.063 \\
JAK1 & rs12405556 & 0.323 & 0.925 \\
& rs17127107 & 1.032 & 0.533 \\
JAK2 & rs10889502 & 1.253 & 0.389 \\
STAT3 & rs7849191 & 0.129 & 0.992 \\
SHP-2 & rs9891119 & 0.780 & 0.680 \\
PPARGC1A & rs4767860 & 0.914 & 0.599 \\
& rs2970847 & 0.163 & 0.999 \\
PRKAA1 & rs6821591 & 0.527 & 0.830 \\
PRKAA2 & rs249429 & 0.975 & 0.556 \\
PRKAB2 & rs3805486 & 1.078 & 0.496 \\
PRKAG1 & rs1342382 & 0.647 & 0.780 \\
PRKAG2 & rs6588640 & 0.867 & 0.620 \\
& rs6937 & 1.671 & 0.191 \\
PRKAG3 & rs3766522 & 1.625 & 0.186 \\
Alpha-MSH & rs10783299 & 0.748 & 0.710 \\
NPY & rs5017427 & 1.593 & 0.226 \\
\hline & rs9648724 & 0.083 & 0.997 \\
& rs645163 & 0.643 & 0.774 \\
& rs6436094 & 0.558 & 0.832 \\
& & 0.589 & 0.816 \\
& & 2.323 & 0.045 \\
\hline & & & \\
& & &
\end{tabular}

The haplotype in LD block was analyzed by using SNPStats software. The analysis results are shown in Table 9. The haplotype of LD block in each gene was not found to be statistically significant.

3.6. SNP-SNP Interaction Results. We analyzed 23 SNPS of 15 genes under the pathways of the LEP pathway by logistic regression model-based SNP-SNP interaction analysis, and the partial results are shown in Table 10. According to the test level of $0.01,2$ pairs of statistically significant results were obtained. The OR value of the interaction term is 1.311, which corresponds to the interaction between rs16147 and rs1044471, and the corresponding genes are NPY and ADIPOR2. The OR value of the interaction term is 1.422 , which corresponds to the interaction between rs2167270 and rs5435, and the corresponding genes are LEP and SLC2A4.

We uploaded 8 genes from the LEP signaling pathway to the STRING (Search Tool for the Retrieval of Interacting Genes/Proteins) tool. The interaction between proteins encoded by these genes was analyzed, and the results are shown in Figure 2.

3.7. Pathway Analysis Result. Four kinds of kernel functions linear, linear.weighted, IBS, and IBS.weighted were used to analyze the SNP set based on pathways. The results are shown in Table 11 . No statistical significance was found whether covariates were added or not
$(P>0.05)$, nor was the empirical $P$ value obtained by bootstrap method.

\section{Discussion}

The prevalence of diabetes increases significantly in recent decades, affecting about $6 \%$ of adult population globally. Therefore, it is one of the major health care challenges in the world [26]. In China, the incidence of T2D in adults has been increasing over recent decades [27]. T2DM is a major chronic disease that is affected by genetic and environmental factors [28]. The development of T2DM involves multiple factors and signaling pathways, such as hyperglycemia, dyslipidemia, and oxidative damage. The main pathological features are insulin resistance and damage to the function of cells, which is a chronic metabolic disease caused by the interaction of genetic and environmental factors [29, 30].

Heredity has all along been mentioned to play an important role in the development of diabetes [31]. Genome-wide association studies have identified more than 100 independent SNPS associated with T2DM regulation and risk [32]. Several gene polymorphisms of leptin have been reported to be associated with T2DM [2, 33]. For example, the leptin (LEP) G2548A polymorphism has been associated with increased leptin production and plasma secretion from adipocytes [34]. Human LEP is located on chromosome7q31.3, and its translational product is leptin, which plays a decisive role in the regulation of human appetite and results in severe metabolic disorders [35]. Such signalization upregulates and downregulates genes involved in cell differentiation, proliferation, apoptosis, and synthesis of extracellular matrix proteins [36].

In this study, rs2167270 and rs16147 were associated with type 2 diabetes in the unit-point correlation analysis. However, in order to further confirm its association with type 2 diabetes, independent sample verification is needed in Chinese Han population. Linkage disequilibrium analysis and correlation analysis based on single type times get within the eight genes of the pathway between the two respective loci; there is a linkage disequilibrium relationship; each genotype in LD block of haploid analysis has not been having a positive result. The LEP pathway gene SNP-SNP interactions analysis results have statistical significance and illustrate that the SNP-SNP interactions (gene interactions) are part of the genetic structure of type 2 diabetes mellitus. LEP gene SNP in pathway, the statistically significant results in the occurrence of type 2 diabetes, one of more than just a channel or certain genes, plays an important role, may also involve two pathways even based on the joint action of multiple pathways between LEP pathways of SNP set correlation analysis, and has statistically significant results. Considering that a few SNPS were selected in each gene, it is necessary to increase the number of SNPS in follow-up studies.

However, the sample of this study is only the Han population in Guangdong Province. The research group has certain limitations. It is necessary to further verify in other 







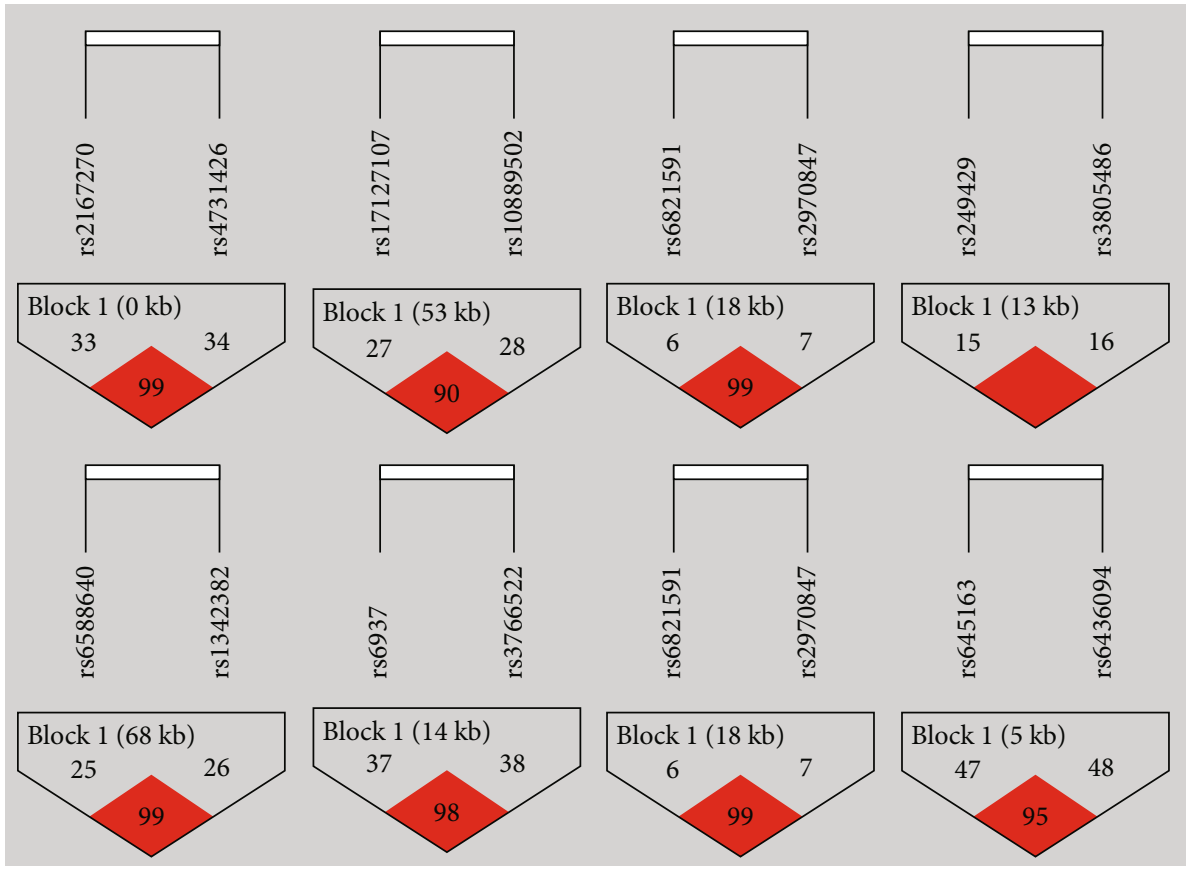

FIGURE 1: Results of linkage disequilibrium analysis of 8 genes in the LEP signaling pathway.

TABLE 9: Results of haplotype unconditional logistic regression analysis of 8 genes in LD block in the LEP signaling pathway.

\begin{tabular}{|c|c|c|c|c|c|c|c|}
\hline Gene & SNP & & SNP & & Freq & OR $(95 \%$ CI $)$ & $P$ \\
\hline \multirow{3}{*}{ LEP } & \multirow{3}{*}{ rs2167270 } & G & \multirow{3}{*}{ rs4731426 } & $\mathrm{C}$ & 0.695 & 1.00 & - \\
\hline & & $\mathrm{A}$ & & G & 0.238 & $1.14(0.99-1.32)$ & 0.076 \\
\hline & & G & & G & 0.067 & $0.99(0.77-1.27)$ & 0.921 \\
\hline \multirow{4}{*}{ JAK1 } & \multirow{4}{*}{ rs 10889502} & $\mathrm{C}$ & \multirow{4}{*}{ rs17127107 } & $\mathrm{C}$ & 0.352 & 1.00 & - \\
\hline & & G & & $\mathrm{C}$ & 0.333 & $1.09(0.94-1.27)$ & 0.261 \\
\hline & & $\mathrm{C}$ & & G & 0.304 & $1.07(0.91-1.25)$ & 0.411 \\
\hline & & G & & G & 0.011 & $1.57(0.74-3.37)$ & 0.240 \\
\hline \multirow{3}{*}{ PPARGC1A } & \multirow{3}{*}{ rs2970847 } & $\mathrm{C}$ & \multirow{3}{*}{ rs6821591 } & $\mathrm{T}$ & 0.673 & 1.00 & - \\
\hline & & $\mathrm{T}$ & & $\mathrm{C}$ & 0.238 & $1.00(0.86-1.15)$ & 0.950 \\
\hline & & $\mathrm{C}$ & & $\mathrm{C}$ & 0.087 & $0.95(0.76-1.19)$ & 0.674 \\
\hline \multirow{3}{*}{ PRKAA1 } & \multirow{3}{*}{ rs249429 } & $\mathrm{T}$ & \multirow{3}{*}{ rs3805486 } & A & 0.546 & 1.00 & - \\
\hline & & $\mathrm{T}$ & & G & 0.234 & $1.01(0.87-1.18)$ & 0.881 \\
\hline & & $\mathrm{C}$ & & A & 0.221 & $1.05(0.90-1.23)$ & 0.532 \\
\hline \multirow{3}{*}{ PRKAA2 } & \multirow{3}{*}{ rs 1342382} & $\mathrm{~T}$ & \multirow{3}{*}{ rs6588640 } & G & 0.496 & 1.00 & - \\
\hline & & A & & G & 0.253 & $0.95(0.82-1.11)$ & 0.531 \\
\hline & & $\mathrm{T}$ & & $\mathrm{A}$ & 0.250 & $0.93(0.80-1.07)$ & 0.300 \\
\hline \multirow{3}{*}{ PRKAB2 } & \multirow{3}{*}{ rs3766522 } & $\mathrm{A}$ & \multirow{3}{*}{ rs6937 } & $\mathrm{C}$ & 0.541 & 1.00 & - \\
\hline & & A & & $\mathrm{T}$ & 0.340 & $1.05(0.92-1.21)$ & 0.481 \\
\hline & & $\mathrm{T}$ & & $\mathrm{T}$ & 0.118 & $1.14(0.93-1.39)$ & 0.212 \\
\hline \multirow{3}{*}{ PRKAG2 } & \multirow{3}{*}{ rs2970847 } & $\mathrm{C}$ & \multirow{3}{*}{ rs6821591 } & $\mathrm{T}$ & 0.673 & 1.00 & - \\
\hline & & $\mathrm{T}$ & & $\mathrm{C}$ & 0.238 & $1.00(0.86-1.15)$ & 0.951 \\
\hline & & $\mathrm{C}$ & & $\mathrm{C}$ & 0.087 & $0.95(0.76-1.19)$ & 0.673 \\
\hline \multirow{3}{*}{ PRKAG3 } & \multirow{3}{*}{ rs6436094 } & $\mathrm{A}$ & \multirow{3}{*}{ rs645163 } & $\mathrm{C}$ & 0.433 & 1.00 & - \\
\hline & & G & & $\mathrm{T}$ & 0.422 & $1.02(0.89-1.16)$ & 0.801 \\
\hline & & G & & $\mathrm{C}$ & 0.137 & $0.95(0.79-1.15)$ & 0.621 \\
\hline
\end{tabular}


TABLE 10: Significant results of SNP-SNP interaction of genes in the LEP signaling pathway.

\begin{tabular}{lccccccc}
\hline CHR1 & SNP1 & CHR2 & SNP2 & OR_INT $*$ & $\chi^{2}$ & $P$ & Genes \\
\hline 7 & rs16147 & 12 & rs1044471 & 1.311 & 8.390 & 0.004 & NPY $\times$ ADIPOR2 \\
7 & rs2167270 & 17 & rs5435 & 1.422 & 9.870 & 0.002 & LEP $\times$ SLC2A4 \\
\hline
\end{tabular}

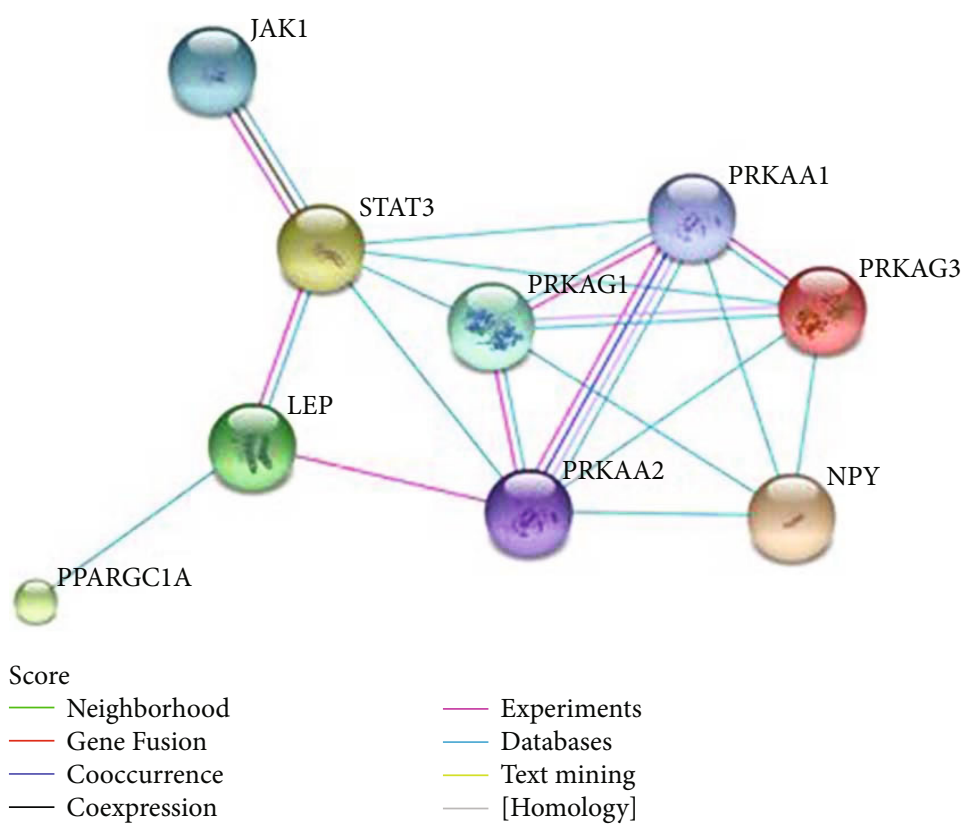

FIgURE 2: The interaction map of 8 genes in the LEP signaling pathway.

TABLE 11: SNP set analysis results based on the LEP pathway.

\begin{tabular}{lcccc}
\hline Model & Kernel & Q & $P$ & Resampling $P$ \\
\hline \multirow{3}{*}{ Without covariates } & Linear & 2072.896 & 0.673 & 0.116 \\
& Linear.weighted & 211.471 & 0.684 & 0.672 \\
& IBS & 73.692 & 0.134 & 0.115 \\
\hline \multirow{3}{*}{ With covariates } & IBS.weighted & 103.078 & 0.631 & 0.124 \\
& Linear & 2132.481 & 0.114 & 0.641 \\
& Linear.weighted & 211.693 & 0.603 & 0.620 \\
\hline
\end{tabular}

populations to provide new clues for the prevention and treatment strategy of T2DM.

\section{Conclusion}

In summary, we found that rs2167270 and rs16147 may interact to affect the risk of T2DM but remained to further confirm their association, and independent sample validation is required in the Chinese Han population. According to the logistic kernel machine regression results, we cannot think the LEP signaling pathway is associated with T2DM.

\section{Data Availability}

The data used to support the findings of this study are available from the corresponding author upon request.

\section{Conflicts of Interest}

The authors declared no conflicts of interest.

\section{Authors' Contributions}

All the authors made substantial contributions to the conception and design. Haibing $\mathrm{Yu}$, Lin $\mathrm{Xu}$, Hao Liu, Jialu Huang, 
Yuanlin Ding, and Danli Kong collected the data. Ling Luo, Rong Chen, Jian xu, Chunwen Lin, Weiying Chen, Yuxun Xie, and Huihuang Yang made substantial contributions to the analysis and interpretation of data. Haibing Yu wrote the first draft of the manuscript. All the authors were involved in revising the manuscript critically for important intellectual content. Yuanlin Ding and Danli Kong gave the final approval of the version to be published. All the authors read and approved the final manuscript.

\section{Acknowledgments}

We appreciate all authors for their contributions and physicians and participants. The study is supported by the Discipline Construction Project of Guangdong Medical University (Fund No. 4SG21264P), the Basic and Applied Basic Research Foundation of Guangdong Province Regional Joint Fund Project (the Key Project, Fund No. 2020B1515120021), the 2021 Guangdong Provincial Medical Research Fund Project (Fund No. A2021395), the Natural Science Foundation of Guangdong Province Basic and Applied Basic Research Fund (Fund No. 2021A1515010061), the 2020 Zhanjiang City Science and Technology Development Special Fund Competitive Allocation Project (Fund No. 2020A01031), the 2020 Guangdong Medical University Scientific Research Fund Natural Science Key Cultivation Project (Fund No. GDMUZ2020008), the 2019 Guangdong Medical University College Student Innovation Experimental Project (Key Project, Fund No. ZZDG003), the 2019 Characteristic Innovation Projects (Natural Science) of Ordinary Universities in Guangdong Province (Fund No. 2019KTSCX047), the 2020 Dongguan Science and Technology Commissioner Project, and the Young Innovative Talents Projects in Ordinary Universities in Guangdong Province (Fund No. 2018KQNCX088).

\section{References}

[1] L. Zhang, Y. Qin, D. Liang et al., "Association of polymorphisms in LEPR with type 2 diabetes and related metabolic traits in a Chinese population," Lipids in Health and Disease, vol. 17, no. 1, p. 2, 2018.

[2] A. Teleginski, M. Welter, H. R. Frigeri et al., "Leptin (rs7799039) and solute carrier family 30 zinc transporter (rs13266634) polymorphisms in Euro-Brazilian pregnant women with gestational diabetes," Genetics and Molecular Research, vol. 16, no. 1, p. 16(1), 2017.

[3] Y. Liu, S. Q. Chen, Z. H. Jing et al., "Association of LEPR Gln223Arg polymorphism with T2DM: a meta-analysis," Diabetes Research and Clinical Practice, vol. 109, no. 3, pp. e21e26, 2015.

[4] K. Rehman, M. S. H. Akash, and Z. Alina, "Leptin: a new therapeutic target for treatment of diabetes mellitus," Journal of Cellular Biochemistry, vol. 119, no. 7, pp. 5016-5027, 2018.

[5] R. Taylor, "Insulin resistance and type 2 diabetes," Diabetes, vol. 61, no. 4, pp. 778-779, 2012.

[6] C. Capurso and A. Capurso, "From excess adiposity to insulin resistance: the role of free fatty acids," Vascular Pharmacology, vol. 57, no. 2-4, pp. 91-97, 2012.
[7] J. Y. Kim, J. H. Sohn, J. H. Lee, and J. W. Park, "Obesity increases airway hyperresponsiveness via the TNF- $\alpha$ pathway and treating obesity induces recovery," PLoS One, vol. 10, no. 2, article e0116540, 2015.

[8] M. S. H. Akash, K. Rehman, and A. Liaqat, "Tumor necrosis factor-alpha: role in development of insulin resistance and pathogenesis of type 2 diabetes mellitus," Journal of Cellular Biochemistry, vol. 119, no. 1, pp. 105-110, 2018.

[9] B. Salgin, K. K. Ong, A. Thankamony, P. Emmett, N. J. Wareham, and D. B. Dunger, "Higher fasting plasma free fatty acid levels are associated with lower insulin secretion in children and adults and a higher incidence of type 2 diabetes," The Journal of Clinical Endocrinology and Metabolism, vol. 97, no. 9, pp. 3302-3309, 2012.

[10] Y. Zhang, R. Proenca, M. Maffei, M. Barone, L. Leopold, and J. M. Friedman, "Positional cloning of the mouse_obese_gene and its human homologue," Nature, vol. 372, no. 6505, pp. 425-432, 1994.

[11] F. H. Lau, K. Vogel, J. P. Luckett et al., "Sandwiched white adipose tissue: a microphysiological system of primary human adipose tissue," Tissue Engineering. Part C, Methods, vol. 24, no. 3, pp. 135-145, 2018.

[12] A. Yadav, M. A. Kataria, V. Saini, and A. Yadav, "Role of leptin and adiponectin in insulin resistance," Clinica Chimica Acta, vol. 417, pp. 80-84, 2013.

[13] A. E. Achari and S. K. Jain, "Adiponectin, a therapeutic target for obesity, diabetes, and endothelial dysfunction," International Journal of Molecular Sciences, vol. 18, no. 6, p. 1321, 2017.

[14] J. Y. Oh, "Regional adiposity, adipokines, and insulin resistance in type 2 diabetes," Diabetes and Metabolism Journal, vol. 36, no. 6, pp. 412-414, 2012.

[15] M. Sambataro, G. Perseghin, G. Lattuada, G. Beltramello, L. Luzi, and G. Pacini, "Lipid accumulation in overweight type 2 diabetic subjects: relationships with insulin sensitivity and adipokines," Acta Diabetologica, vol. 50, no. 3, pp. 301-307, 2013.

[16] A. C. Könner and J. C. Brüning, "Selective insulin and leptin resistance in metabolic disorders," Cell Metabolism, vol. 16, no. 2, pp. 144-152, 2012.

[17] J. Letonja, M. Završnik, J. Makuc et al., "Sirtuin 1 rs7069102 polymorphism is associated with diabetic nephropathy in patients with type 2 diabetes mellitus," Bosnian Journal of Basic Medical Sciences, 2021.

[18] S. Zhang, J. Xu, D. Cui et al., "Genotype distribution of CNDP1 polymorphisms in the healthy Chinese Han population: association with $\mathrm{HbAlc}$ and fasting blood glucose," Journal Diabetes Research, vol. 2020, article 3838505, 2020.

[19] M. Cui, Y. Gao, Y. Zhao et al., "Association between adiponectin gene polymorphism and environmental risk factors of type 2 diabetes mellitus among the Chinese population in Hohhot," BioMed Research International, vol. 2020, Article ID 6383906, 2020.

[20] Z. Zhu, Y. Zhang, R. Bai et al., "Association of genetic polymorphisms in microRNAs with type 2 diabetes mellitus in a Chinese population," Frontiers in Endocrinology, vol. 11, article 587561, 2021.

[21] S. M. Khan, N. El Karte, C. S. El Hajj et al., "Association between type 2 diabetes mellitus \& TCF7L2 gene variants in the Emirati population: genetics of diabetes in the United Arab Emirates," American Journal of Human Biology, vol. 33, no. 1, article e23434, 2021. 
[22] U. Juttada, S. Kumpatla, R. Parveen, and V. Viswanathan, "TCF7L2 polymorphism a prominent marker among subjects with type-2-diabetes with a positive family history of diabetes," International Journal of Biological Macromolecules, vol. 159, pp. 402-405, 2020.

[23] V. Bains, H. Kaur, and B. Badaruddoza, “Association analysis of polymorphisms in LEP (rs7799039 and rs2167270) and LEPR (rs1137101) gene towards the development of type 2 diabetes in North Indian Punjabi population," Gene, vol. 754, article 144846, 2020.

[24] Y. Cui, G. Li, S. Li, and R. Wu, "Designs for linkage analysis and association studies of complex diseases," Methods in Molecular Biology, vol. 620, p. 219, 2010.

[25] B. K. C. Chan, "Applied human genetic epidemiology," Advances in Experimental Medicine and Biology, vol. 1082, pp. 145-216, 2018.

[26] M. M. Yang, J. Wang, J. J. Fan et al., "Variations in the obesity gene "LEPR" contribute to risk of type 2 diabetes mellitus: evidence from a meta-analysis," Journal of Diabetes Research, vol. 2016, 12 pages, 2016.

[27] Y. Tian, J. Xu, T. Huang et al., "A novel polymorphism (rs35612982) in CDKAL1 is a risk factor of type 2 diabetes: a case-control study," Kidney and Blood Pressure Research, vol. 44, no. 6, pp. 1313-1326, 2019.

[28] R. M. Altall, S. Y. Qusti, N. Filimban et al., "SLC22A1 And ATM Genes Polymorphisms Are Associated With The Risk Of Type 2 Diabetes Mellitus In Western Saudi Arabia: A Case-Control Study," The Application of Clinical Genetics, vol. Volume 12, pp. 213-219, 2019.

[29] M. Hivert, J. L. Vassy, and J. B. Meigs, "Susceptibility to type 2 diabetes mellitus-from genes to prevention," Nature Reviews Endocrinology, vol. 10, no. 4, pp. 198-205, 2014.

[30] T. Guo, L. Zhu, J. Tan et al., "Promoting effect of triterpenoid compound from Agrimonia pilosa Ledeb on preadipocytes differentiation via up-regulation of PPAR $\gamma$ expression," Pharmacognosy Magazine, vol. 11, no. 41, pp. 219-225, 2015.

[31] M. Mambiya, M. Shang, Y. Wang et al., "The play of genes and non-genetic factors on type 2 diabetes," Frontiers in Public Health, vol. 7, p. 349, 2019.

[32] A. Varshney, L. J. Scott, R. P. Welch et al., "Genetic regulatory signatures underlying islet gene expression and type 2 diabetes," Proceedings of the National Academy of Sciences, vol. 114, no. 9, pp. 2301-2306, 2017.

[33] P. Wu, W. Wen, J. Li et al., "Systematic review and metaanalysis of randomized controlled trials on the effect of SGLT2 inhibitor on blood leptin and adiponectin level in patients with type 2 diabetes," Hormone and Metabolic Research, vol. 51, no. 8, pp. 487-494, 2019.

[34] M. Yang, S. Peng, W. Li, Z. Wan, L. Fan, and Y. du, "Relationships between plasma leptin levels, _leptin_G2548A, _leptin receptor_Gln223Arg polymorphisms and gestational diabetes mellitus in Chinese population," Scientific Reports, vol. 6, no. 1, p. 6, 2016.

[35] Y. Zhao, N. Hong, X. Liu et al., "A novel mutation in leptin gene is associated with severe obesity in Chinese individuals," BioMed Research International, vol. 2014, 3 pages, 2014.

[36] Á. Ramírez, M. Hernández, R. Suárez-Sánchez et al., “Type 2 diabetes-associated polymorphisms correlate with SIRT1 and TGF- $\beta 1$ gene expression," Annals of Human Genetics, vol. 84, no. 2, pp. 185-194, 2020. 